\title{
Drinking Levels and Profiles of Alcohol Addicted Rats Predict Response to Nalmefene
}

\begin{abstract}
Jerome Clifford Foo ${ }^{1 * t}$, Valentina Vengeliene ${ }^{2 t}$, Hamid Reza Noori ${ }^{3}$, Ikuhiro Yamaguchi ${ }^{4}$, Kenji Morita ${ }^{4}$, Toru Nakamura ${ }^{5}$, Yoshiharu Yamamoto ${ }^{4}$ and Rainer Spanagel ${ }^{2 *}$
\end{abstract}

\begin{abstract}
'Department of Genetic Epidemiology in Psychiatry, Central Institute of Mental Health, Medical Faculty Mannheim, University of Heidelberg, Mannheim, Germany, ${ }^{2}$ Institute of Psychopharmacology, Central Institute of Mental Health, Medical Faculty Mannheim, University of Heidelberg, Mannheim, Germany, ${ }^{3}$ Neuronal Convergence Group, Max Planck Institute for Biological Cybernetics, Tübingen, Germany, ${ }^{4}$ Department of Physical and Health Education, Graduate School of Education, The University of Tokyo, Tokyo, Japan, ${ }^{5}$ Biomedical Engineering and Health Informatics Laboratory, Center for Industry-University Collaboration, Graduate School of Engineering Science, Osaka University, Osaka, Japan
\end{abstract}

OPEN ACCESS

Edited by:

Andrew Lawrence,

The Florey Institute of Neuroscience and Mental Health, Australia

Reviewed by:

Selena Bartlett,

Translational Research Institute

Australia

Karen K. Szumlinski,

University of California,

Santa Barbara, United States

*Correspondence:

Jerome Clifford Foo

jerome.foo@zi-mannheim.de

Rainer Spanagel

rainer.spanage/@zi-mannheim.de

†These authors have contributed

equally to this work

Specialty section: This article was submitted to Translational Pharmacology, a section of the journal Frontiers in Pharmacology

Received: 10 February 2019 Accepted: 15 April 2019 Published: 07 May 2019

Citation:

Foo JC, Vengeliene $V$, Noori HR, Yamaguchi I, Morita K, Nakamura T, Yamamoto $Y$ and Spanagel $R$ (2019) Drinking Levels and Profiles of Alcohol Addicted Rats Predict Response to Nalmefene.

Front. Pharmacol. 10:471 doi: 10.3389/fphar.2019.00471
Background: Pharmacotherapeutic options supporting the treatment of alcohol dependence are recommended and available but underutilized, partly due to questions about efficacy. Nalmefene, a $\mu$-opioid receptor antagonist and partial kappa receptor agonist, is recommended for reduction of alcohol consumption, but evidence about its effectiveness has been equivocal; identifying factors which predict response will help optimize treatment.

Methods: The alcohol deprivation effect paradigm is a tightly controlled procedure comprising repeated deprivation and reintroduction phases, leading to increased preference for alcohol; reintroduction approximates relapse. Using a digital drinkometer system measuring high-resolution drinking behavior, we examined the effects of nalmefene on relapse drinking behavior in alcohol addicted rats. We also tested whether drinking behavior in the relapse phase prior to nalmefene administration predicted treatment response. We further examined whether longitudinal drinking behavior and locomotor activity predicted treatment response.

Results: Our results showed that nalmefene $(0.3 \mathrm{mg} / \mathrm{kg})$ reduced relapse-like consumption significantly ( 20\%) compared to vehicle on the first 2 days of alcohol reintroduction. Examining the first $6 \mathrm{~h}$ of a preceded treatment-free relapse episode revealed drinking patterns clustering the rats into responders (reduction of $>40 \%$, $n=17$ ) and non-responders (reduction of $<40 \%, n=7$ ) to subsequent nalmefene treatment. During the first $6 \mathrm{~h}$ of the preceding relapse phase, responders consumed more alcohol than non-responders; the amount of alcohol consumed during each drinking approach was larger but frequency of drinking did not differ. Longitudinal drinking behavior and locomotor activity did not significantly predict response.

Conclusion: Our results suggest that nalmefene reduces alcohol intake during a relapse-like situation but effectiveness can differ greatly at the individual level. However, who responds may be informed by examining drinking profiles and rats that show high drinking levels prior to treatment are more likely to respond to nalmefene. 


\section{INTRODUCTION}

Pharmacotherapeutic options supporting the treatment of alcohol use disorder (AUD) are available and recommended for the management of AUD but are underutilized, in part due to the drugs having modest effects (Litten et al., 2018). Even the first-line treatments, acamprosate, a compound that dampens a hyper-glutamatergic state (Spanagel et al., 2005; Umhau et al., 2010; Spanagel et al., 2014), and naltrexone, a $\mu$-opioid receptor antagonist, have only shown reductions in risk of drinking to $86 \%$ (Rösner et al., 2010a) and 83\% (Rösner et al., 2010b), respectively, of the placebo rate, which does not lead to confidence in drug efficacy, motivating further research aimed at the discovery of new medications.

Nalmefene was recently approved by the European Medicines Agency as a treatment for human adults with AUD who wished to reduce their alcohol consumption but not necessarily abstain (Mann et al., 2016). Nalmefene is also a $\mu$-opioid receptor antagonist as well as partial kappa receptor agonist (Bart et al., 2005), which is thought to have a similar mechanism of action as naltrexone (Osborn et al., 2010). Pre-clinical research has found evidence of nalmefene's effectiveness to significantly reduce dependence-induced alcohol self-administration in rats (June et al., 1998, 2004; Calleja-Conde et al., 2016) and to be more effective than naltrexone in this respect (Walker and Koob, 2008). It is suggested that nalmefene counters alcohol-induced dysregulations of the $\mu$ - and $\kappa$-opioid receptor systems (Nealey et al., 2011).

There is also some clinical evidence for advantages of nalmefene over naltrexone (Soyka, 2016) and beneficial effects of nalmefene on endpoints such as reducing heavy drinking days and total alcohol consumption have been observed (Mann et al., 2016), particularly with an as-needed approach (Gual et al., 2013; Soyka, 2014; Francois et al., 2015). However, concerns have been raised as to the strength of this evidence and questions remain about the clinical efficacy of this drug (Palpacuer et al., 2015; Naudet et al., 2016). Response rates have been reported to vary widely, leaving significance for treatment of individual patients uncertain (Fitzgerald et al., 2016).

The identification of people who are likely to respond to nalmefene would improve treatment decisions, and identifying factors which could predict response will result in better clinical outcomes and could serve to optimize implementation of the as-needed approach (Soyka, 2014). In this respect it is important to note that the level of alcohol consumption at baseline prior to treatment may predict treatment response to nalmefene. Thus, a recent meta-analysis indicates that patients with a low or medium drinking risk level (up to $60 \mathrm{~g}$ alcohol per day) all failed to show any clinically relevant effect vs. placebo whereas nalmefene was modestly effective in patients with high or very high drinking risk levels (>61 g/day) (van den Brink et al., 2018) Furthermore, we have recently shown in a longitudinal preclinical study that specific drinking patterns can predict relapse behavior (Foo et al., 2017). Here we set out in a prospective study to examine if the level of alcohol consumption and drinking patterns prior to treatment are predictive of the effectiveness of nalmefene.
In the present study, we designed a longitudinal preclinical study for testing the hypothesis that drinking levels and patterns are predictive for a nalmefene response. For this purpose we used the alcohol deprivation effect (ADE) model to measure relapse-like behavior. In rats that have long-term voluntary access to alcohol followed by deprivation for several weeks, the re-presentation of alcohol leads to relapse-like drinking - a temporal increase in alcohol intake over the baseline drinking (Vengeliene et al., 2009; Spanagel, 2017). This robust phenomenon is called the ADE. This animal model has been used in numerous preclinical and translational alcohol studies and helped identifying new treatment targets with good predictive validity (Spanagel, 2009; Vengeliene et al., 2009; Spanagel, 2017). In order to precisely assess alcohol consumption and drinking patterns in our here designed prospective study we used a fully automated digital drinkometer system allowing high-resolution capture of drinking data and thus analysis of drinking profiles (Vengeliene et al., 2013). With this system we are also able to identify characteristics of alcohol drinking such as alcohol "liking" or "wanting." An increase in alcohol "wanting" can be measured as an increased frequency of approaches to more concentrated alcohol solutions whereas alcohol "liking" is assessed by the amount of alcohol consumed per drinking approach (Vengeliene et al., 2013, 2015). With different computational approaches (Nakamura et al., 2016; Foo et al., 2017) we tested whether individual drinking levels and patterns prior to treatment are associated with nalmefene efficacy.

\section{MATERIALS AND METHODS}

\section{Animals}

In the experiment 1 , sixteen two-month old male Wistar rats were used, and in the experiment 2, twenty-four two-month old male Wistar rats (all from the breeding colony at the $\mathrm{CIMH}$, Mannheim, Germany) were used. All animals were housed individually in standard rat cages (Eurostandard Type III; Ehret, Emmendingen, Germany) under an artificial $12 \mathrm{~h} / 12 \mathrm{~h}$ light/dark cycle (lights on at 7 a.m.). Room temperature was kept constant (temperature: $23 \pm 1{ }^{\circ} \mathrm{C}$, humidity: $55 \pm 5 \%$ ). Standard laboratory rat food (Ssniff, Soest, Germany) and tap water were provided ad libitum throughout the experiment. Rat body weights were measured weekly. All experiments were conducted in accordance with the ethical guidelines for the care and use of laboratory animals, and were approved by the local animal care committee (Regierungspräsidium Karlsruhe, Germany).

\section{Drugs}

Alcohol drinking solutions were prepared from 96\% ethanol (Sigma-Aldrich, Germany) and then diluted with tap water. Nalmefene (Lundbeck, Denmark) was dissolved in $0.9 \%$ saline. The solution was freshly prepared and injected as a volume of $1 \mathrm{ml} / \mathrm{kg}$ subcutaneously (s.c.). Control experiments were performed following administration of saline. 


\section{Drinkometer System}

The experiments were performed using a computer-monitored Drinkometer system (TSE Systems, Bad Homburg, Germany), which enables continuous long-term monitoring of liquid consumption by amount and time in a standard rat home cage (Eurostandard Type III). The system is equipped with four drinking stations to allow liquid choice. Each drinking station consists of a glass vessel containing the liquid and a high precision sensor for weighing the amount of liquid removed from the glass vessel. Spillage and evaporation are minimized by using special bottle caps. The whole system is mounted to a custom-made free-swinging steel frame in order to avoid any environmental disturbances (see also Vengeliene et al., 2013). The weight of each vessel is measured in $200 \mathrm{~ms}$ steps and saved in $1 \mathrm{~s}$ steps, and ultra-high resolution changes in volume are detected down to $0.01 \mathrm{~g}$. For experiment 1, the weight of drinking vessels was recorded in 5-min intervals, and for experiment 2, the sampling interval was set at 1-min, giving per minute values of solutions consumed.

\section{Long-Term Voluntary Alcohol Consumption With Repeated Deprivation Phases}

The ADE is a tightly controlled experimental procedure used to model excessive relapse-like drinking in rodents (Vengeliene et al., 2013). The procedure begins with a long-term (8 weeks) baseline period of voluntary alcohol consumption in a fourbottle free-choice paradigm in which rats are continuously presented with water and three different concentrations of ethanol (5, 10 and 20\%). This baseline period is followed by a two-week deprivation period, after which alcohol is reintroduced and the ADE, which is characterized by robust increases in alcohol intake and preference for stronger solutions, is observed. Subsequent deprivation (2 weeks long) and reintroduction phases (4-6 weeks long) are randomly introduced, resulting in an increased preference for alcohol. Thus, drinking patterns during the $\mathrm{ADE}$ represent an important target for understanding both relapse mechanisms and investigating effects of drug treatment.

\section{Locomotor Activity Measurements}

Rat locomotor activity was monitored by use of an infrared sensor connected to a Mouse-E-Motion recording and data storing system (Infra-e-motion, Henstedt-Ulzburg, Germany). The device was placed above each cage so that the rat could be detected at any position inside the cage. The device sampled every second and the sensor could detect rat body movements at least $1.5 \mathrm{~cm}$ from one sample point to the successive one. For the experiment 1, monitoring of locomotor activity started 4 days before the drug treatment procedure and was continued for two more post-treatment days. The percentage of each rat's locomotor activity during and after treatment days was calculated by using the "before treatment" activity data as a reference. For the experiment 2, locomotor activity was measured continuously and recorded every minute during the initial 8-week baseline drinking period, as well as first, second, fifth and sixth ADE periods (see also Foo et al., 2017).

\section{Experiment 1}

Nalmefene treatment was introduced at the end of the 8th alcohol deprivation. In order to study the effects of nalmefene, rats were divided into two groups $(n=8)$ in such way that the mean baseline intake of water and 5, 10, and 20\% of alcohol solutions was approximately the same in each group. Baseline drinking was monitored daily for one week. After the last day of baseline measurement, the alcohol bottles were removed from the cages leaving the animals with free access to food and water for 19 days. Thereafter, each animal was subjected to a total of 5 s.c. injections (starting at 7 p.m. with $12 \mathrm{~h}$ intervals) of either vehicle or nalmefene $(0.3 \mathrm{mg} / \mathrm{kg}$, please note that a prior experiment employing the same experimental paradigm using 0.01 and $0.1 \mathrm{mg} / \mathrm{kg}$ did not produce significant effects, this data is not shown). The alcohol bottles were reintroduced after the second drug administration (at $\sim 9$ a.m. on the 20th day of alcohol deprivation). Each rat's body weight was recorded $24 \mathrm{~h}$ before the first injection and $12 \mathrm{~h}$ after the last injection.

\section{Experiment 2}

In order to explore whether or not the response to nalmefene could be predicted by certain drinking patterns or behavior, we conducted a second experiment with a larger sample and higher resolution assessments, examining the ADE prior to nalmefene administration. In this experiment, nalmefene was injected s.c. to all rats at the end of the 6th two-week long alcohol deprivation. Each animal was subjected to a total of 5 s.c. injections (starting at 7 p.m. with $12 \mathrm{~h}$ intervals) of nalmefene $(0.3 \mathrm{mg} / \mathrm{kg})$. Similarly to the first experiment, the alcohol bottles were reintroduced after the second injection. Each rat's body weight was recorded $24 \mathrm{~h}$ before the first injection and $12 \mathrm{~h}$ after the last injection.

\section{Data Analysis}

Data on total daily ethanol intake, water intake and locomotor activity from experiment 1 was analyzed using a two-way analysis of variance (ANOVA) with repeated measures (factors were: between subjects - treatment group, and within subjects - day). For the analysis of locomotor activity, only the data from the dark phases was used. Whenever significant differences were found, post hoc Student Newman-Keuls test was performed. Data analysis regarding the effects of treatment on the change in the rat body weight was performed using either a one-way ANOVA or independent two-tailed $t$-test.

The effects of nalmefene from experiment 2 were first examined comparing the first 3 days of the 5th (ADE5) and 6th (ADE6) ADEs. Two-way ANOVA with repeated measures was used. The first $6 \mathrm{~h}$ after reintroduction of alcohol were analyzed in greater detail as this is the period where the strongest ADE occurs. This data was analyzed using two-way ANOVA with repeated measures (factors were: between subjects - ADE, and within subjects - time). To quantify response to nalmefene treatment, total alcohol consumption (intake of pure ethanol in $\mathrm{g}$ per $\mathrm{kg}$ of body weight in $6 \mathrm{~h}$ ) was compared across ADE5 and ADE6 to give a \% reduction score: Response $=\left(\right.$ Consumption ${ }_{\mathrm{ADE} 5}-$ Consumption $_{\mathrm{ADE} 6}$ )/Consumption $\mathrm{ADE}_{5}$. After examining the response distribution it was found that two clusters emerged 
above and below the mean. We thus used the mean to classify response of rats to nalmefene: those below the mean response level were classified as "Non-responders" and those above it were classified as "Responders." To test whether established drinking levels and profiles existing prior to nalmefene administration could inform response to nalmefene, we calculated during ADE5 during the first $6 \mathrm{~h}$ of alcohol re-exposure: (Litten et al., 2018) total alcohol intake (Spanagel et al., 2005) approach frequency (i.e., average number of approaches) for each alcohol solution and (Umhau et al., 2010) approach size (i.e., amount of alcohol consumed per drinking approach). To characterize the effects of nalmefene in Responders and Non-responders, we also looked at the drinking profiles in ADE6. Comparisons between Responders and Non-responders were made using Welch's ANOVA given unequal sample sizes. The chosen level of significance was $p<0.05$.

Longitudinal locomotor activity was examined after classification of Responders and Non-Responders. It was examined whether locomotor activity patterns differed between the two groups during ADE5 and ADE6, as well as during initial baseline and deprivation periods during which addiction-like behavior was established. Following our previous work (Foo et al., 2017) we examined whether weekly activity, representing stable locomotor patterns (6day intervals, not including the day when experimenters entered the room), was informative with respect to response status to nalmefene. Based on per minute activity counts, we calculated local statistics for locomotor activity over the week for each rat. Mean per minute activity, as well as variance and skewness of the weekly locomotor distribution were examined. Differences in mean activity could indicate psychomotor agitation/retardation, while variance characterizes variability of activity and skewness represents intermittency of activity; shown to be biomarkers able to characterize disease states (Kim et al., 2013; Nakamura et al., 2016). Furthermore, circadian amplitude was also calculated for the weekly locomotor data. Briefly, the continuous wavelet transform, which can be used to characterize periodic patterns, was applied to the weekly locomotor data. The power of the continuous wavelet coefficient for the frequency 1 cycle/day, or circadian amplitude, was extracted (see Foo et al., 2017 for further detail). Repeated measures ANOVA was used to test for differences between longitudinal group trajectories.

\section{RESULTS}

\section{Experiment 1}

Following the re-introduction of alcohol solutions after a period of abstinence, the vehicle treated group showed a typical increase in alcohol consumption, indicating the occurrence of an ADE. This increase was not different from that observed during the first deprivation periods (data not shown). Hence, a two-way ANOVA for repeated measures revealed a general increase in alcohol intake after an eight deprivation phase as compared to basal drinking [factor day: $F(6,84)=41.8$, $p<0.0001]$ (Figure 1A). Analysis of data also showed
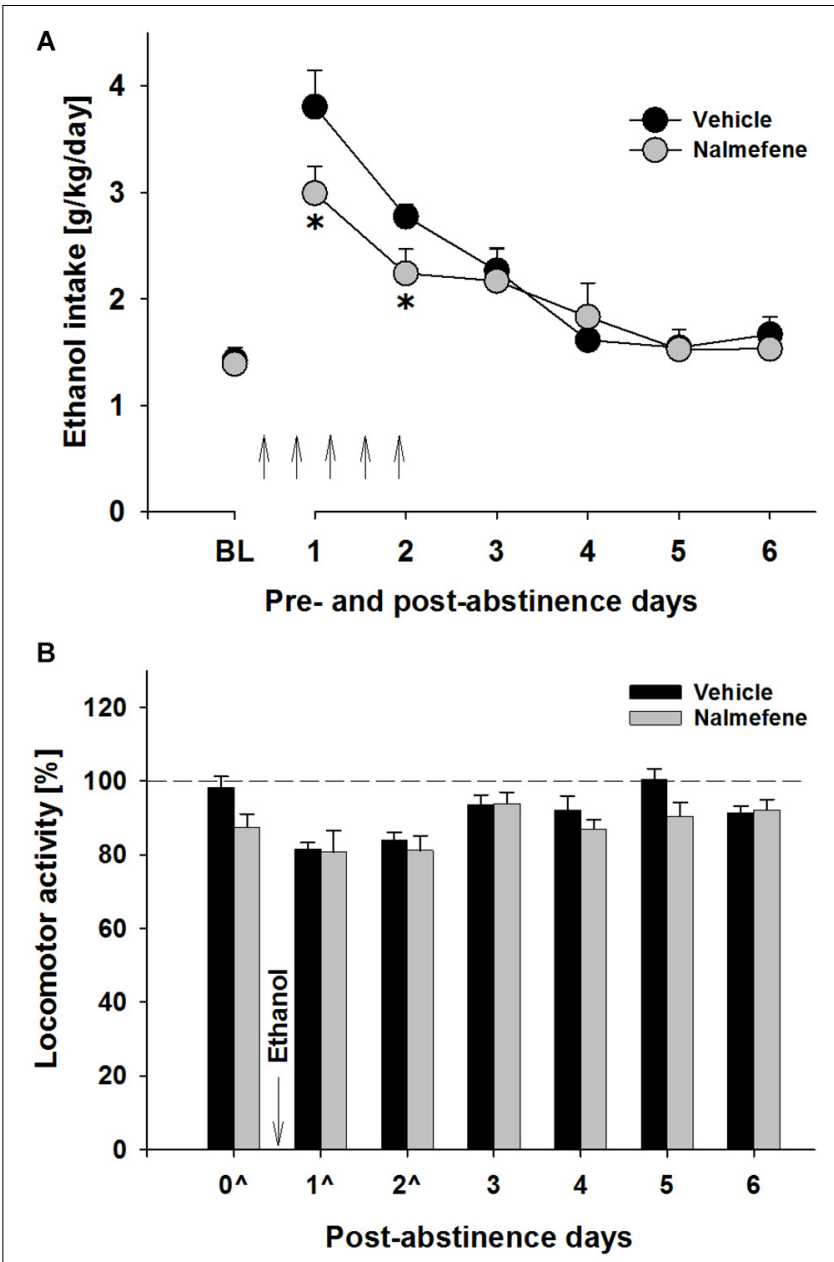

FIGURE 1 | Total ethanol intake ( $\mathrm{g} / \mathrm{kg} /$ day) before and after an alcohol deprivation period of 3 weeks $(\mathbf{A})$ and locomotor activity of the last abstinence day and the first post-abstinence days (B). Arrows indicate the administration of vehicle $(n=8)$ and $0.3 \mathrm{mg} / \mathrm{kg}$ of nalmefene $(n=8)$. A total of 5 injections were given (starting at 7 p.m. with $12 \mathrm{~h}$ intervals), the first injection was given $12 \mathrm{~h}$ before post-abstinence drinking begun. The average of the last 6 days measurements of ethanol intake is presented as the baseline drinking (BL). Locomotor activity is shown as 12-h post-injection intervals of the animals' active phase. The percentage of each rat's locomotor activity during and after treatment days was calculated with respect to basal activity prior to treatment (dashed line). Data are presented as means \pm S.E.M. *indicates significant difference from the vehicle control group, $p<0.05$.

that nalmefene treatment significantly reduced alcohol intake during the first post-abstinence days when compared to intake by vehicle treated animals [factor treatment group $\times$ day interaction effect: $F(6,84)=2.5, p<0.05]$. This treatment did not cause loss of body weight, however, animals from the nalmefene treatment group did not gain as much body weight as the vehicle treated rats [vehicle: $+1.4 \%$ and nalmefene: $+0.7 \%$, factor treatment group $t(14)=2.5, p<0.05]$. Stronger doses might increase response, but may also result in unwanted effects. Nalmefene treatment had no effect on either water intake [factor treatment group $\times$ day interaction effect: $p=0.84$ ] (data not shown) or home-cage activity 
of rats measured as total number of movements during their active phase [factor treatment group $\times$ day interaction effect: $p=0.71$ ] (Figure 1B), demonstrating that this drug is well tolerated.

\section{Experiment 2}

When comparing the first 3 days of the regular $\mathrm{ADE}$ period (ADE5) preceding the $\mathrm{ADE}$ period with nalmefene administration (ADE6 - all 24 animals received s.c. nalmefene injections), it was observed that for each day and for the whole 3 day period, rats consumed less total alcohol during ADE6 [factor ADE: $F(1,46)=15.5, p<0.001$ and factor $\mathrm{ADE} \times$ time interaction effect: $F(3,138)=3.6, p<0.05]$ (Figure 2A). While statistically significant, this effect was modest (i.e., an average of $18.6 \%$ decrease). As mentioned above, to better quantify the drinking behavior, we also analyzed drinking during the first $6 \mathrm{~h}$ of the ADE, since during the first hours after alcohol reintroduction the ADE is the largest with effects tapering off quickly over time (Vengeliene et al., 2009, 2013; Foo et al., 2017). Hence, the overall decrease of hourly consumption between ADE5 and

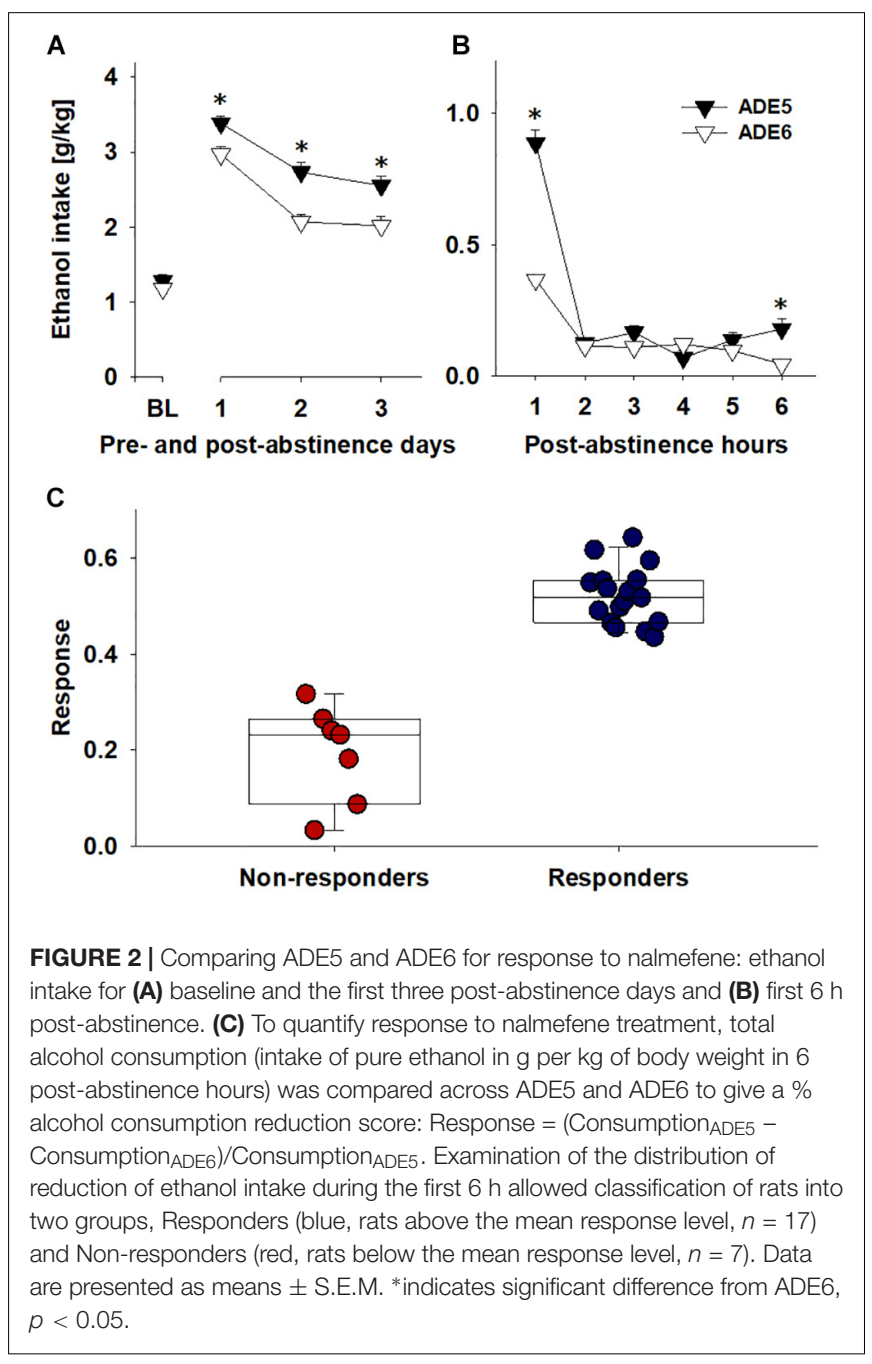

ADE6 was significantly different [factor ADE: $F(1,46)=81.0$, $p<0.0001]$, and this difference was most pronounced during the first hour of alcohol re-exposure [factor ADE $\times$ time interaction effect: $F(5,230)=28.6, p<0.0001$ ] (Figure 2B). The average Response (i.e., a \% reduction score described in Data Analysis) during the first $6 \mathrm{~h}$ was $42.6 \%$. After examining the distribution of responses for all rats, the rats were classified into two groups: those below the mean were classified as "Non-responders" and those above it were classified as "Responders" (Figure 2C).

\section{Drinking Profiles During ADE5}

Drinking behavior during ADE5 was analyzed to see whether drinking levels and patterns of rats could predict effects of nalmefene. Examining consumption patterns in Responders and Non-Responders during ADE5 revealed that overall, responders had significantly higher alcohol consumption levels during the first $6 \mathrm{~h}$ of ADE5 $[F(1,20.5)=39.8, p<0.001]$ (Figure 3A). This effect was solely driven by higher consumption of $20 \%$ alcohol during this time $[F(1,20.9)=16.5, p<0.001]$. Intake of 5 and $10 \%$ alcohol did not differ between Responders and Non-responders (Figure 3A). Frequency of approaches to the alcohol bottles - which we have used as an indication of "alcohol wanting” in our previous work (Vengeliene et al., 2013, 2015) did not differ significantly between groups neither for each alcohol solutions nor for alcohol accesses in total (Figure 3B). Average total alcohol approach size was larger in Responders $[F(1,21.5)=6.6, p<0.05]$, an effect which appears to have been driven by higher approach size of $20 \%$ solution $[F(1,18.6)=6.6$, $p<0.05]$ (Figure 3C). The amount of alcohol that is consumed during a drinking approach has been defined in our previous studies as an indication for "alcohol liking" (Vengeliene et al., 2013, 2015). This finding leads to the conclusion that nalmefene responders show higher preceding drinking levels which are mainly driven by alcohol liking.

\section{Drinking Profiles During ADE6 (Nalmefene Treatment)}

During the first hours of ADE6, total alcohol intake during the first $6 \mathrm{~h}$ after alcohol re-exposure in Responders was lower than that in Non-responders $[F(1,12.9)=6.9, p<0.05]$ (data not shown). This appears to have been a result of an additive effect across solutions; although all solutions showed reduced consumption, individual solution approach size did not statistically significantly differ $(5 \%: p=0.41 ; 10 \%: p=0.21 ; 20 \%$ : $p=0.30)$. No significant effects of nalmefene were observed on approach frequency $[p=0.73]$ (Figures 3D-F).

\section{Longitudinal Locomotor Activity}

Recently, we were able to show that alterations in locomotor activity patterns; especially, instability of circadian rhythms (Foo et al., 2017), can be predictive of future relapse behavior. Therefore, we also examined locomotor activity patterns for Responders and Non-Responders. Descriptively, comparison of longitudinal circadian amplitude trajectories was suggestive of differences between Responders and Non-responders, but this main effect did not reach significance (i.e., $p=0.18$ ) (Figure 4A). For mean, variance and skewness measures of locomotor activity 


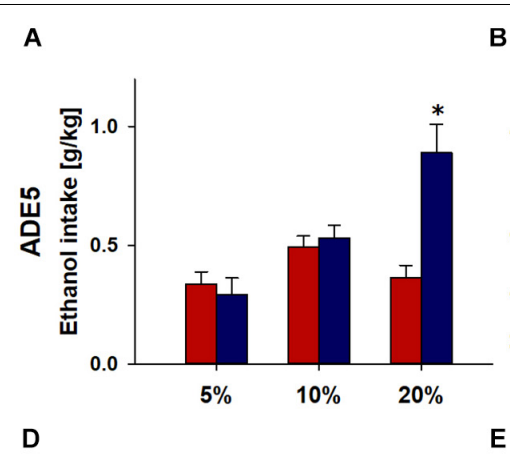

D

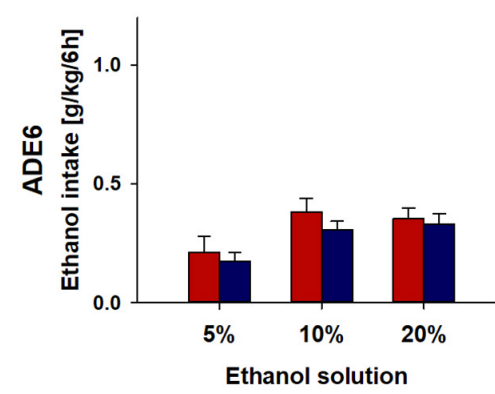

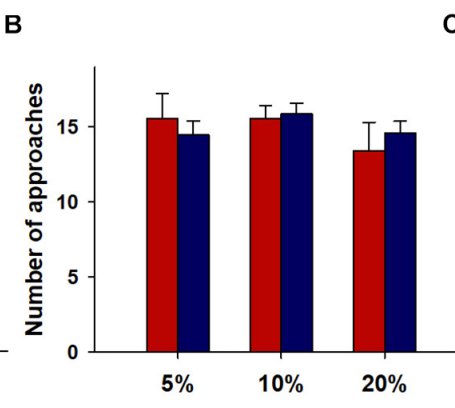

E

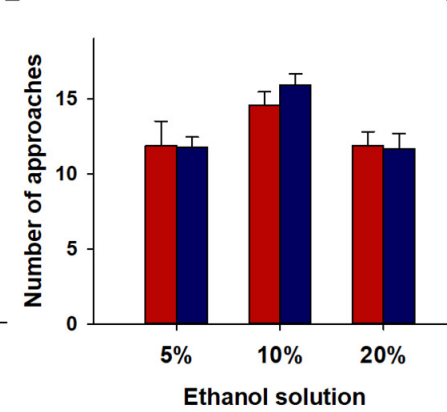

C

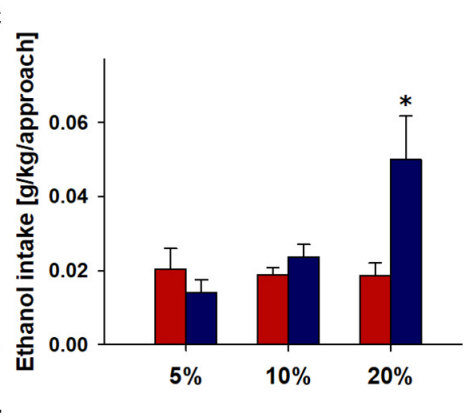

F

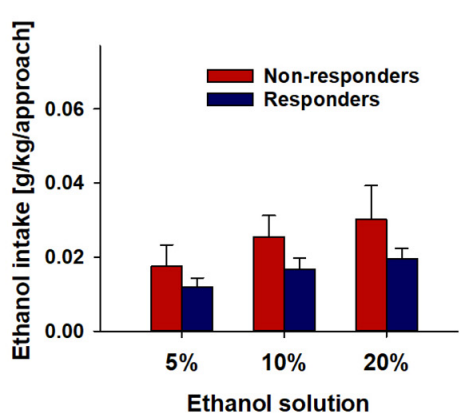

FIGURE 3 | Drinking profiles of rats $(n=24)$ during the first 6 post-abstinence hours of ADE5 (A-C) and ADE6 (D-F). Repeated administration of 0.3 mg/kg of nalmefene was performed during ADE6. Total ethanol intake (A,D), number of approaches (B,E) and amount of ethanol consumed per approach (C,F) for each alcohol solution is shown in rats above the mean response level (Responders, $n=17$ ) and below the mean response level (Non-Responders, $n=7$ ) to nalmefene treatment. Data are presented as means \pm S.E.M. *indicates significant difference from Non-responders, $p<0.05$.

patterns again no differences were observed between Responders and Non-responders (Figures 4B-D).

\section{DISCUSSION}

Our study demonstrated that nalmefene treatment reduced relapse-like alcohol consumption in male rats during the first post-abstinence days in a four-bottle free-choice setting. Nalmefene was the most effective in rats which consumed greater amounts of highly concentrated alcohol per drinking approach prior to drug treatment. Nalmefene treatment did not change water intake and locomotor activity compared to vehicle treatment, demonstrating the selectivity of the tested compound toward alcohol consumption.

The in vivo pharmacology of nalmefene is suggested to be similar to naltrexone (Osborn et al., 2010; Spanagel and Vengeliene, 2013), acting on opioid receptors and it shares the ability to reduce the subjective "high" feeling after alcohol consumption (i.e., "alcohol liking") (Drobes et al., 2004; Hendershot et al., 2017). In the present experiments, the effects of nalmefene (reduction of approximately $20 \%$ in alcohol consumption) were comparable with that of other opioid antagonists given under similar experimental conditions (Hölter and Spanagel, 1999).

Like other medications for relapse prevention, nalmefene is only modestly effective with many patients failing to benefit from this treatment. Especially, inter-individual variability may play a role for the effectiveness of nalmefene (Fitzgerald et al., 2016).
As such, it is important to identify potential high responders, so that the maximum benefits can be derived from the drug. The distribution of response to nalmefene in our study suggests a role for inter-individual differences; while most rats experienced reductions in alcohol consumption (ranging from 3.4 to $64.2 \%$ in the first $6 \mathrm{~h}$ of $\mathrm{ADE}$ ) as a result of treatment, rats were also clearly separable into better and poorer responding groups. The results of the present investigation suggest that drinking profiles and alcohol consumption levels may be able to inform drug response. We observed that those rats that exhibited more pronounced response to nalmefene had consumed more alcohol, especially of a higher concentration, during the preceding "regular ADE," potentially suggesting that better responders have a stronger preference for alcohol. This finding on the preclinical level is in line with a recent meta-analysis that indicates that patients with a low or medium drinking risk level (up to $60 \mathrm{~g}$ alcohol per day) all failed to show any clinically relevant effect vs. placebo, whereas nalmefene was modestly effective in patients with high or very high drinking risk levels (>61 g/day) (van den Brink et al., 2018). Thus, individuals that show high baseline alcohol consumption levels prior treatment are more likely to respond to nalmefene. This is an important conclusion, since patients that have high or even very high drinking risk levels are the ones who experience the most severe health consequences (Rehm et al., 2018).

We also observed that the effects of nalmefene were driven by reduction of alcohol consumption per drinking approach, while no reductions in frequency of approaches to alcohol bottles were observed. Thus, our results appear to inform the distinction between "liking" and "wanting," which has been an important 
topic in the field of addiction (Robinson and Berridge, 2003; Spanagel, 2009). Finding that approach size during a drinking episode, but not frequency was reduced during ADE supports the idea of a separation of "wanting" and "liking" (Berridge and Aldridge, 2008; Vengeliene et al., 2013, 2015), which is consistent with the idea that the neural systems responsible for ethanol "wanting" are different from those that mediate the hedonic "liking" effects of alcohol (Spanagel, 2009); and drug treatments may differentially target these mechanisms. For example, it has been observed that at higher levels of alcohol craving, drinking was reduced at a significantly greater rate with naltrexone as compared to acamprosate (Richardson et al., 2008). The present results support the idea that the mechanism of action of nalmefene is on the hedonic value of ethanol; while rats continue to approach the bottles with the same frequency, they drink less as the alcohol is rendered less appetitive by the drug. This is in line with recent neuroimaging evidence of a nalmefene-caused reduction in "reward anticipation" in striatal regions (Quelch et al., 2017). This hedonic "liking," is thought to be governed by opioid neurotransmission in the rostro-dorsal quarter of the medial nucleus accumbens shell (Pecina, 2008).

In terms of longitudinal locomotor activity, descriptively, the measure of circadian amplitude suggested that responders

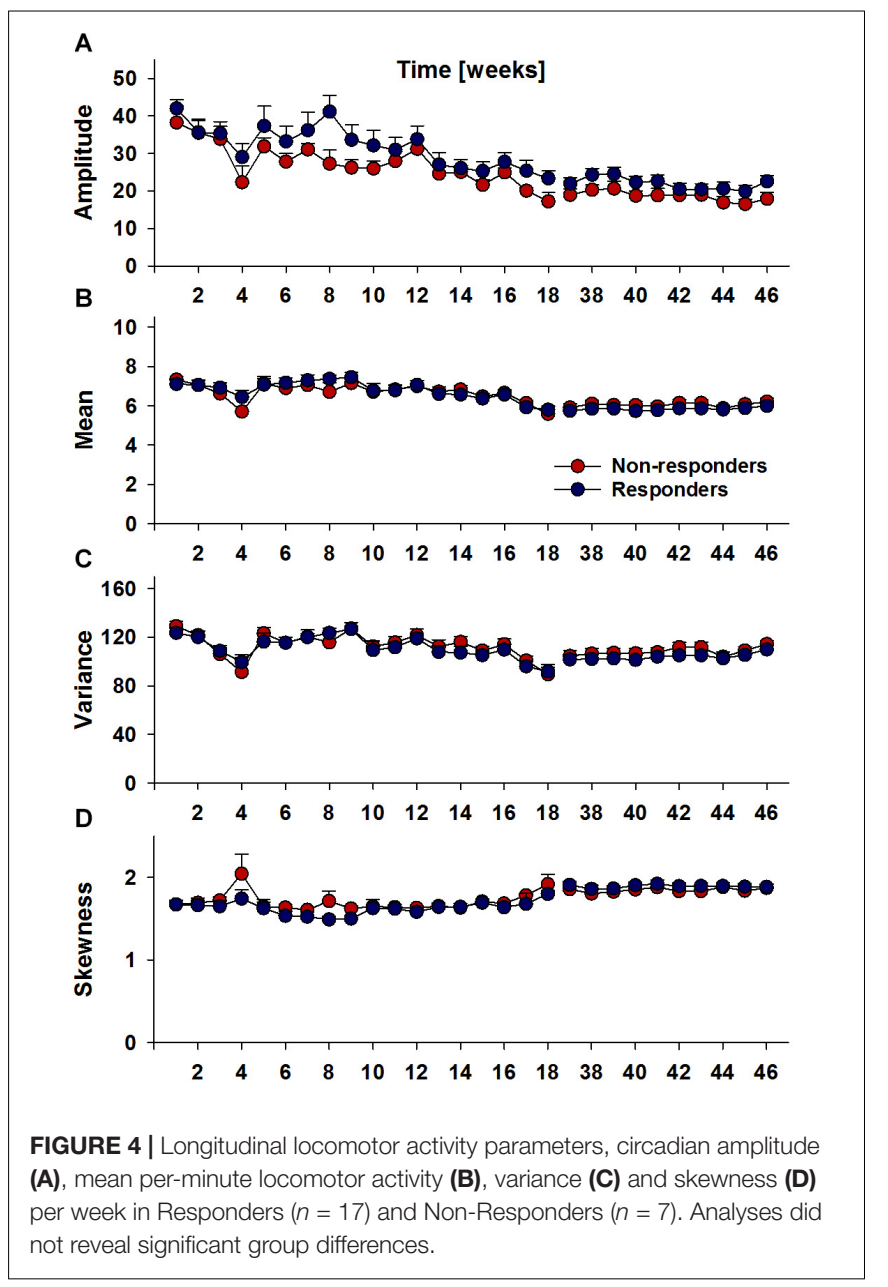

may have higher circadian amplitudes than non-responders, but these group differences did not reach significance. These analyses did not yield evidence of overarching longitudinal differences between groups; differences may not extend to long-term behavioral patterns and may be restricted to local relapse periods.

It should be recognized that due to the efficacy of the drug, the number of responders and non-responders were necessarily unbalanced; larger sample sizes will be needed to further test these results, and it is expected that increased power will improve the ability of these approaches to identify differences between groups, informing drug treatment response. It should also be noted that the present definition of response to nalmefene is based on short-term effects at a specific dosage. Long-term administration may reveal different response patterns. It is possible that not only response but also sensitivity is reflected in the present findings, and further research is needed to clearly delineate underlying mechanisms. Determining the relationship between ethanol consumption and effects on opioid systems may lead to improved target selection and the development of a different class of opioid drugs.

The present findings were made possible through the use of a drinkometer which acquired continuous longitudinal data. In humans, it is difficult to reach the time resolution and accuracy offered by the drinkometer and efficacy measures such as quantity and frequency of drinking have thus far only been acquired in a comparatively limited fashion (much research examines abstinence rates and or number of binge days using retrospective self-reports or other cross-sectional methods). In the emerging era of Information and Communication Technologies (ICT), acquisition of this data is becoming possible using mobile and wearable technologies and an ambulatory assessment approach (Trull and Ebner-Priemer, 2013), and is likely to be key to further characterize treatment response and help to better identify and quantify effective treatment time courses. Furthermore, to improve translatability of findings to clinical applications, research needs to be extended in the direction of including female animals.

Prospective clinical studies are now needed to test if the drinking profiles identified here and alcohol drinking risk levels are indeed predictive for the efficacy of nalmefene. If "baseline drinking profiles" can be assessed through the detailed and rigorous collection of data in clinical settings is expected to be important to inform the targeted use of not only nalmefene in the recommended "harm-reduction," "as needed" approach, but other drugs and types of treatments.

\section{AUTHOR CONTRIBUTIONS}

RS and VV designed the study, interpreted the findings, and wrote the manuscript. JF analyzed the data, interpreted the findings, and wrote the manuscript. VV performed the experiments and analyzed the data. HN, IY, KM, TN, and YY analyzed the locomotor activity data. All authors approved the final version of the manuscript. 


\section{FUNDING}

This work was supported by the Bundesministerium für Bildung und Forschung (e:Med program FKZ: 01ZX1503 and 01ZX1311A (Spanagel et al., 2013) and AERIAL (EE1406C) and the

\section{REFERENCES}

Bart, G., Schluger, J. H., Borg, L., Ho, A., Bidlack, J. M., and Kreek, M. J. (2005). Nalmefene induced elevation in serum prolactin in normal human volunteers: partial kappa opioid agonist activity? Neuropsychopharmacology 30, 2254-2262. doi: 10.1038/sj.npp.1300811

Berridge, K. C., and Aldridge, J. W. (2008). Decision utility, the brain, and pursuit of hedonic goals. Soc. Cogn. 26, 621-646. doi: 10.1521/soco.2008.26.5.621

Calleja-Conde, J., Echeverry-Alzate, V., Gine, E., Buhler, K. M., Nadal, R., Maldonado, R., et al. (2016). Nalmefene is effective at reducing alcohol seeking, treating alcohol-cocaine interactions and reducing alcohol-induced histone deacetylases gene expression in blood. Br. J. Pharmacol. 173, 2490-2505. doi: $10.1111 /$ bph.13526

Drobes, D. J., Anton, R. F., Thomas, S. E., and Voronin, K. (2004). Effects of naltrexone and nalmefene on subjective response to alcohol among nontreatment-seeking alcoholics and social drinkers. Alcohol. Clin. Exp. Res. 28, 1362-1370. doi: 10.1097/01.alc.0000139704.88862.01

Fitzgerald, N., Angus, K., Elders, A., de Andrade, M., Raistrick, D., Heather, N., et al. (2016). Weak evidence on nalmefene creates dilemmas for clinicians and poses questions for regulators and researchers. Addiction 111, 1477-1487. doi: 10.1111/add.13438

Foo, J. C., Noori, H. R., Yamaguchi, I., Vengeliene, V., Cosa-Linan, A., Nakamura, T., et al. (2017). Dynamical state transitions into addictive behaviour and their early-warning signals. Proc. Biol. Sci. 284:20170882. doi: $10.1098 /$ rspb.2017.0882

Francois, C., Rahhali, N., Chalem, Y., Sorensen, P., Luquiens, A., and Aubin, H. J. (2015). The effects of as-needed nalmefene on patient-reported outcomes and quality of life in relation to a reduction in alcohol consumption in alcoholdependent patients. PLoS One 10:e129289. doi: 10.1371/journal.pone.0129289

Gual, A., He, Y., Torup, L., van den Brink, W., Mann, K., and Group, E. S. (2013). A randomised, double-blind, placebo-controlled, efficacy study of nalmefene, asneeded use, in patients with alcohol dependence. Eur. Neuropsychopharmacol. 23, 1432-1442. doi: 10.1016/j.euroneuro.2013.02.006

Hendershot, C. S., Wardell, J. D., Samokhvalov, A. V., and Rehm, J. (2017). Effects of naltrexone on alcohol self-administration and craving: meta-analysis of human laboratory studies. Addict. Biol. 22, 1515-1527. doi: 10.1111/adb.12425

Hölter, S. M., and Spanagel, R. (1999). Effects of opiate antagonist treatment on the alcohol deprivation effect in long-term ethanol-experienced rats. Psychopharmacology 145, 360-369. doi: 10.1007/s00213005 1069

June, H. L., Cummings, R., Eiler, W. J. II, Foster, K. L., McKay, P. F., Seyoum, R., et al. (2004). Central opioid receptors differentially regulate the nalmefeneinduced suppression of ethanol- and saccharin-reinforced behaviors in alcoholpreferring (P) rats. Neuropsychopharmacology 29, 285-299. doi: 10.1038/sj.npp. 1300338

June, H. L., Grey, C., Warren-Reese, C., Durr, L. F., Ricks-Cord, A., Johnson, A., et al. (1998). The opioid receptor antagonist nalmefene reduces responding maintained by ethanol presentation: preclinical studies in ethanol-preferring and outbred wistar rats. Alcohol. Clin. Exp. Res. 22, 2174-2185. doi: 10.1111/j. 1530-0277.1998.tb05931.x

Kim, J., Nakamura, T., Kikuchi, H., Sasaki, T., and Yamamoto, Y. (2013). Covariation of depressive mood and locomotor dynamics evaluated by ecological momentary assessment in healthy humans. PLoS One 8:e74979. doi: 10.1371/ journal.pone.0074979

Litten, R. Z., Falk, D. E., Ryan, M. L., Fertig, J., and Leggio, L. (2018). Advances in pharmacotherapy development: human clinical studies. Handb. Exp. Pharmacol. 248, 579-613. doi: 10.1007/164_2017_79

Mann, K., Torup, L., Sorensen, P., Gual, A., Swift, R., Walker, B., et al. (2016). Nalmefene for the management of alcohol dependence: review on its pharmacology, mechanism of action and meta-analysis on its clinical efficacy.
ERANET grant "PsiAlc" (01EW1908). We acknowledge financial support by Deutsche Forschungsgemeinschaft within the funding program TRR265 (A05) and Open Access Publishing, by the Baden-Württemberg Ministry of Science, Research and the Arts and by Ruprecht-Karls-Universität Heidelberg.

Eur. Neuropsychopharmacol. 26, 1941-1949. doi: 10.1016/j.euroneuro.2016. 10.008

Nakamura, T., Kiyono, K., Wendt, H., Abry, P., and Yamamoto, Y. (2016). Multiscale analysis of intensive longitudinal biomedical signals and its clinical applications. Proc. IEEE 104, 242-261. doi: 10.1109/jproc.2015.2491979

Naudet, F., Fitzgerald, N., and Braillon, A. (2016). Nalmefene for alcohol dependence: a nice decision? Lancet Psychiatry 3, 1104-1105. doi: 10.1016/ s2215-0366(16)30356-x

Nealey, K. A., Smith, A. W., Davis, S. M., Smith, D. G., and Walker, B. M. (2011). Kappa-opioid receptors are implicated in the increased potency of intra-accumbens nalmefene in ethanol-dependent rats. Neuropharmacology 61, 35-42. doi: 10.1016/j.neuropharm.2011.02.012

Osborn, M. D., Lowery, J. J., Skorput, A. G., Giuvelis, D., and Bilsky, E. J. (2010). In vivo characterization of the opioid antagonist nalmefene in mice. Life Sci. 86, 624-630. doi: 10.1016/j.lfs.2010.02.013

Palpacuer, C., Laviolle, B., Boussageon, R., Reymann, J. M., Bellissant, E., and Naudet, F. (2015). Risks and benefits of nalmefene in the treatment of adult alcohol dependence: a systematic literature review and meta-analysis of published and unpublished double-blind randomized controlled trials. PLoS Med. 12:e1001924. doi: 10.1371/journal.pmed.1001924

Pecina, S. (2008). Opioid reward 'liking' and 'wanting' in the nucleus accumbens. Physiol. Behav. 94, 675-680. doi: 10.1016/j.physbeh.2008.04.006

Quelch, D. R., Mick, I., McGonigle, J., Ramos, A. C., Flechais, R. S. A., Bolstridge, M., et al. (2017). Nalmefene reduces reward anticipation in alcohol dependence: an experimental functional magnetic resonance imaging study. Biol. Psychiatry 81, 941-948. doi: 10.1016/j.biopsych.2016.12.029

Rehm, J., Guiraud, J., Poulnais, R., and Shield, K. D. (2018). Alcohol dependence and very high risk level of alcohol consumption: a life-threatening and debilitating disease. Addict. Biol. 23, 961-968. doi: 10.1111/adb.12646

Richardson, K., Baillie, A., Reid, S., Morley, K., Teesson, M., Sannibale, C., et al. (2008). Do acamprosate or naltrexone have an effect on daily drinking by reducing craving for alcohol? Addiction 103, 953-959. doi: 10.1111/j.1360-0443. 2008.02215.x

Robinson, T. E., and Berridge, K. C. (2003). Addiction. Annu. Rev. Psychol. 54, 25-53. doi: 10.1146/annurev.psych.54.101601.145237

Rösner, S., Hackl-Herrwerth, A., Leucht, S., Lehert, P., Vecchi, S., and Soyka, M. (2010a). Acamprosate for alcohol dependence. Cochrane Database Syst. Rev. CD004332. doi: 10.1002/14651858.CD004332.pub2

Rösner, S., Hackl-Herrwerth, A., Leucht, S., Vecchi, S., Srisurapanont, M., and Soyka, M. (2010b). Opioid antagonists for alcohol dependence. Cochrane Database Syst. Rev. 9:CD001867. doi: 10.1002/14651858.CD001867.pub2

Soyka, M. (2014). Nalmefene for the treatment of alcohol dependence: a current update. Int. J. Neuropsychopharmacol. 17, 675-684. doi: 10.1017/ S1461145713001284

Soyka, M. (2016). Nalmefene for the treatment of alcohol use disorders: recent data and clinical potential. Expert Opin. Pharmacother. 17, 619-626. doi: 10.1517/ 14656566.2016.1146689

Spanagel, R. (2009). Alcoholism: a systems approach from molecular physiology to addictive behavior. Physiol. Rev. 89, 649-705. doi: 10.1152/physrev.00013.2008

Spanagel, R. (2017). Animal models of addiction. Dialogues Clin. Neurosci. 19, 247-258.

Spanagel, R., Durstewitz, D., Hansson, A., Heinz, A., Kiefer, F., Köhr, G., et al. (2013). A systems medicine research approach for studying alcohol addiction. Addict. Biol. 18, 883-896. doi: 10.1111/adb.12109

Spanagel, R., Pendyala, G., Abarca, C., Zghoul, T., Sanchis-Segura, C., Magnone, M. C., et al. (2005). The clock gene Per2 influences the glutamatergic system and modulates alcohol consumption. Nat. Med. 11,35-42. doi: 10.1038/nm1163

Spanagel, R., and Vengeliene, V. (2013). New pharmacological treatment strategies for relapse prevention. Curr. Top. Behav. Neurosci. 13, 583-609. doi: 10.1007/ 7854_2012_205 
Spanagel, R., Vengeliene, V., Jandeleit, B., Fischer, W. N., Grindstaff, K., Zhang, X., et al. (2014). Acamprosate produces its anti-relapse effects via calcium. Neuropsychopharmacology 39, 783-791. doi: 10.1038/npp. 2013.264

Trull, T. J., and Ebner-Priemer, U. (2013). Ambulatory assessment. Annu. Rev. Clin. Psychol. 9, 151-176. doi: 10.1146/annurev-clinpsy-050212-185510

Umhau, J. C., Momenan, R., Schwandt, M. L., Singley, E., Lifshitz, M., Doty, L., et al. (2010). Effect of acamprosate on magnetic resonance spectroscopy measures of central glutamate in detoxified alcohol-dependent individuals: a randomized controlled experimental medicine study. Arch. Gen. Psychiatry. 67, 1069-1077. doi: 10.1001/archgenpsychiatry.2010.125

van den Brink, W., Addolorato, G., Aubin, H. J., Benyamina, A., Caputo, F., Dematteis, M., et al. (2018). Efficacy and safety of sodium oxybate in alcohol-dependent patients with a very high drinking risk level. Addict. Biol. 23, 969-986. doi: 10.1111/adb.12645

Vengeliene, V., Celerier, E., Chaskiel, L., Penzo, F., and Spanagel, R. (2009). Compulsive alcohol drinking in rodents. Addict. Biol. 14, 384-396. doi: 10.1111/ j.1369-1600.2009.00177.x

Vengeliene, V., Noori, H. R., and Spanagel, R. (2013). The use of a novel drinkometer system for assessing pharmacological treatment effects on ethanol consumption in rats. Alcohol. Clin. Exp. Res. 37, E322-E328. doi: 10.1111/j 1530-0277.2012.01874.x

Vengeliene, V., Noori, H. R., and Spanagel, R. (2015). Activation of melatonin receptors reduces relapse-like alcohol consumption. Neuropsychopharmacology 40, 2897-2906. doi: 10.1038/npp.2015.143

Walker, B. M., and Koob, G. F. (2008). Pharmacological evidence for a motivational role of kappa-opioid systems in ethanol dependence. Neuropsychopharmacology 33, 643-652. doi: 10.1038/sj.npp.1301438

Conflict of Interest Statement: The authors declare that the research was conducted in the absence of any commercial or financial relationships that could be construed as a potential conflict of interest.

Copyright (c) 2019 Foo, Vengeliene, Noori, Yamaguchi, Morita, Nakamura, Yamamoto and Spanagel. This is an open-access article distributed under the terms of the Creative Commons Attribution License (CC BY). The use, distribution or reproduction in other forums is permitted, provided the original author(s) and the copyright owner(s) are credited and that the original publication in this journal is cited, in accordance with accepted academic practice. No use, distribution or reproduction is permitted which does not comply with these terms. 